\title{
Hausdorff dimension of scale-sparse Weierstrass-type functions
}

by

\author{
Abel Carvalho (Aveiro)
}

\begin{abstract}
The aim of this paper is to calculate (deterministically) the Hausdorff dimension of the scale-sparse Weierstrass-type functions $W_{s}(x):=\sum_{j \geq 1} \rho^{-\gamma^{j} s} g\left(\rho^{\gamma^{j}} x+\theta_{j}\right)$, where $\rho>1, \gamma>1$ and $0<s<1$, and $g$ is a periodic Lipschitz function satisfying some additional appropriate conditions.
\end{abstract}

1. Introduction. Functions with a uniform fractal structure on the domain occur everywhere in nature, and they are widely applicable in physics because their graphs play an important role as invariant sets in dynamical systems. Examples of this are the Weierstrass-type functions

$$
W_{s}(x):=\sum_{j \geq 1} \rho^{-j s} \cos \left(\rho^{j} x+\theta_{j}\right), \quad x \in \mathbb{R},
$$

where $\rho>1,0<s<1$ and $\theta_{j} \in \mathbb{R}$. The parameter $s$ measures smoothness, more precisely the continuous real function $W_{s}$ belongs to the Hölder class $C^{s}(\mathbb{R})$. Other distinguished parameters of the graphs of continuous real functions on the real line are the box and Hausdorff dimensions. They fall between 1 and 2 because the domain is $\mathbb{R}$ but the graphs are contained in $\mathbb{R}^{2}$. The box dimension of the graph of the function $W_{s}$ is given by the simple and well-known formula $\operatorname{dim}_{\mathrm{B}} \Gamma\left(W_{s}\right)=2-s$.

Nevertheless, the determination of the Hausdorff dimension for graphs of such functions remains an open problem even for the typical case $\rho=2$ and $\theta_{j}=0$. Hunt [4] stated (see Theorem 3.1 below) that the Hausdorff dimension of $\Gamma\left(W_{s}\right)$ equals $2-s$ for almost all sequences $\left(\theta_{j}\right)_{j \in \mathbb{N}}$, but this result is not deterministic. In the present work, however, we will deal with the far easier scale-sparse Weierstrass-type functions

2010 Mathematics Subject Classification: 28A80, 28A78.

Key words and phrases: Hausdorff dimension, measures and $d$-sets, Weierstrass functions. 


$$
W_{s}(x):=\sum_{j \geq 1} \rho^{-\gamma^{j} s} \cos \left(\rho^{\gamma^{j}} x+\theta_{j}\right)
$$

Theorem 3.2 below is the main result of the paper and gives deterministically the Hausdorff dimension for these functions. The necessary definitions are given in Section 2 and the proofs are shifted to Section 4. Strictly positive constants are represented by $c_{\text {indices }}$ or $c, c^{\prime}, \ldots$

2. Some definitions in fractal geometry. We introduce the concepts of box and Hausdorff dimensions, measures and $d$-sets.

Definition 2.1. Let $x:=\left(x_{1}, \ldots, x_{n}\right) \in \mathbb{R}^{n}$. Then $|x|:=\sqrt{\sum_{i=1}^{n} x_{i}^{2}}$ is the Euclidean norm of $x$. Let $\emptyset \neq U \subset \mathbb{R}^{n}$. Then the diameter of $U$ is $|U|:=\sup _{x, y \in U}|x-y|$.

Definition 2.2. A measure on $\mathbb{R}^{n}$ is a function defined on the Borel subsets of $\mathbb{R}^{n}$, satisfying $\mu(\emptyset)=0, \mu(U) \leq \mu(U \cup V)$, and $\mu\left(\bigcup_{k \in \mathbb{N}} U_{k}\right)=$ $\sum_{k \in \mathbb{N}} \mu\left(U_{k}\right)$ for disjoint collections. (We will consider only mass distributions with $\mu\left(\mathbb{R}^{n}\right)=1$.)

The paper deals mainly with Hausdorff dimension, $\operatorname{dim}_{\mathrm{H}}$, though upper and lower box dimensions, $\overline{\operatorname{dim}}_{\mathrm{B}}$ and $\underline{\operatorname{dim}}_{\mathrm{B}}$, are also invoked; for definitions, see [3, pp. 25-41].

Remark 2.3 ([3, pp. 43 and 55]). Let $\emptyset \neq E \subset \mathbb{R}^{n}$. Then $0 \leq \operatorname{dim}_{\mathrm{H}} E \leq$ $\underline{\operatorname{dim}}_{\mathrm{B}} E \leq \overline{\operatorname{dim}}_{\mathrm{B}} E \leq n$. Let $B_{r}$ be balls (of radius $r$ ) centered at $P \in E=$ $\operatorname{supp} \mu$; if $r^{d} \geq c \mu\left(B_{r}\right)$ for $0<r<1$ then $\overline{\operatorname{dim}}_{\mathrm{B}} E \geq d$ (and the respective result for $\leq$ also holds).

Definition 2.4. Consider $d>0$ and let $\emptyset \neq E \subset \mathbb{R}^{n}$. Then $E$ is a $d$-set if there is a mass distribution $\mu$ on $\mathbb{R}^{n}$ such that $\mu\left(B_{r}\right) \approx_{r} r^{d}$, i.e. $c_{1} r^{d} \leq \mu\left(B_{r}\right) \leq c_{2} r^{d}$ for all balls $B_{r}$ with $0<r<1$ and centered at any $P \in E=\operatorname{supp} \mu$.

By Remark 2.3, $\operatorname{dim}_{\mathrm{H}} E=\operatorname{dim}_{\mathrm{B}} E=d$. (However, box and Hausdorff dimensions can behave distinctly - cf. first table of Theorem 4.3 of [2].)

3. Hausdorff dimension of graphs. In the present section we deal with the box and Hausdorff dimensions of the graphs of Weierstrass-type functions, particularly in the scale-sparse case for which we give in Theorem 3.2 deterministically the exact value for the Hausdorff dimension.

Theorem 3.1 (Theorem 1 of [4], with elementary adaptations). Consider $\rho>1,0<s<1$, and let $\theta:=\left(\theta_{j}\right)_{j \in \mathbb{N}} \subset[0,2 \pi]$. Let $W_{s}:[0,1] \rightarrow \mathbb{R}$ be defined by $W_{s}(x):=\sum_{j \geq 1} \rho^{-j s} \cos \left(\rho^{j} x+\theta_{j}\right)$. Then

$$
\operatorname{dim}_{\mathrm{H}} \Gamma\left(W_{s}\right)=2-s \quad \text { for almost all sequences } \theta \text {, }
$$


where the set of all sequences $\theta, \amalg:=[0,2 \pi]^{\mathbb{N}}$, is endowed with the measure induced by the Lebesgue (uniform) measure normalized by the factor $(2 \pi)^{-1}$, so $\amalg$ has measure 1. By almost all we mean all $\theta \in \amalg \backslash \amalg^{\prime}$ for some $\amalg^{\prime} \subset \amalg$ with measure zero.

By looking at Theorem 3.1 of [2], we see that the value $2-s$ coincides with the box dimension of $W_{s}$. Moreover, Theorem 3.1 above remains valid if we replace the constant $s$ by any convergent sequence $\left(s_{j}\right)_{j \in \mathbb{N}} \subset \mathbb{R}^{+}$such that $s:=\lim _{j \rightarrow \infty} s_{j}$ satisfies $0<s<1$. As we can see in Theorem 4.3 of [2], referring to [6], if $1 \leq p \leq \infty$ and $0<s<1$ then $2-s$ is the maximal Hausdorff dimension for all continuous real functions with smoothness $s$, so Theorem 3.1 is important in what concerns the existence of functions that achieve that maximal value.

This theorem can also be generalized by replacing the cosine function by a more general real, Lipschitz and non-constant periodic function $g$ satisfying some additional hypotheses (see Theorem 2 of [4]). One inconvenience is that it is not deterministic, so even for the typical case $\rho=2$ and $\theta=0$, the Hausdorff dimension of $\Gamma\left(W_{s}\right)$ for the so called Weierstrass function is still unknown. Despite that fact, in [5, p. 800, Theorem 8], it is stated (deterministically) that

$$
\operatorname{dim}_{\mathrm{H}} \Gamma\left(W_{s}\right) \geq 2-s-c / \log \rho,
$$

for all sequences $\theta$, where $c$ does not depend on $\rho$, so the right hand side grows slowly to $2-s$ when $\rho$ tends to infinity. (Recall, on the other hand, the well-known relation $\operatorname{dim}_{\mathrm{H}} \Gamma\left(W_{s}\right) \leq \underline{\operatorname{dim}}_{\mathrm{B}} \Gamma\left(W_{s}\right) \leq \overline{\operatorname{dim}}_{\mathrm{B}} \Gamma\left(W_{s}\right) \leq 2-s$.) Though the proof of the inequality above uses techniques very different from the ones employed in [4], both references use similar measures, respectively induced by Cantor and Lebesgue measures.

As we can see in Remark 3.3(b) below, by using techniques similar to but simpler than the ones employed in [5], we can recover the lower bound $2-s-c / \log \rho$ given in that reference. Moreover, by applying those techniques we prove the deterministic result stated in Theorem 3.2. which gives the Hausdorff dimension of scale-sparse series, coinciding with the lower box dimension.

TheOREM 3.2. Let $g: \mathbb{R} \rightarrow \mathbb{R}$ be a periodic Lipschitz function satisfying $g(x)-g(y) \approx_{x, y} x-y$ for $x$ and $y$ belonging to some subinterval of the real line, and $g(x)-g(y) \approx_{x, y}-(x-y)$ on another subinterval. Consider $\rho, \gamma>1,0<s<1$ and $\left(\theta_{j}\right)_{j \in \mathbb{N}} \subset \mathbb{R}$. Let $W_{s}:[0,1] \rightarrow \mathbb{R}$ be defined by $W_{s}(x):=\sum_{j \geq 1} \rho^{-\gamma^{j} s} g\left(\rho^{\gamma^{j}} x+\theta_{j}\right)$. Then, for any sequence $\left(\theta_{j}\right)_{j \in \mathbb{N}}$, we have the identity

$$
\operatorname{dim}_{H} \Gamma\left(W_{s}\right)=2-\Xi(\gamma, s)=2-\frac{\gamma s}{1-s+\gamma s} .
$$


We observe that for fixed $\gamma>1$, the one-variable function $\Xi(\gamma, \cdot)$ is strictly increasing and ranges over $(0,1)$ for $0<s<1$; analogously, for fixed $0<s<1$, the function $\Xi(\cdot, s)$ is strictly increasing and ranges over $(s, 1)$ for $1<\gamma<\infty$.

Theorem 3.2 is an extension of a result given in [1, which considered the case when $g$ is a pure triangular wave and $\theta_{j}=0$ for all $j \in \mathbb{N}$. Observe that by using Theorem 4.1 of [2], together with Remark 2.3, we can now easily deduce the same value for the lower box dimension:

$$
\underline{\operatorname{dim}}_{\mathrm{B}} \Gamma\left(W_{s}\right)=2-\Xi(\gamma, s)=2-\frac{\gamma s}{1-s+\gamma s}=1+\frac{1-s}{1-s+\gamma s} .
$$

Moreover, an easy estimation of the oscillations of $W_{s}$, by using only standard arguments, shows that $\overline{\operatorname{dim}}_{\mathrm{B}} \Gamma\left(W_{s}\right)=2-s$. Furthermore, Theorems 3.1 and 3.2 , as well as the comments and estimates following them, also hold for the limiting case $s=1$; in other words, $\operatorname{dim}_{\mathrm{H}} \Gamma\left(W_{1}\right)=\operatorname{dim}_{\mathrm{B}} \Gamma\left(W_{1}\right)=1$. This follows from the identity $\overline{\operatorname{dim}}_{\mathrm{B}} \Gamma\left(W_{1}\right)=1$ for this limiting case and Remark 2.3.

As a special case of Theorem 3.2 it follows that, for all $\varkappa>0$ and $0<s<1$, the function of [7, p. 121], when the graph is in $\mathbb{R}^{2}, f(x)=$ $\sum_{j \geq 1} 2^{-\nu_{j} s} \sum_{k \in \mathbb{Z}} \omega\left(2^{\nu_{j}} x-k\right)$, where $\nu_{j}:=2^{j \varkappa}$, actually has Hausdorff dimension $2-\Xi\left(2^{\varkappa}, s\right)$. (See the beginning of Section 5 of [2].) Furthermore, the equalities above give the same value for the lower box dimension, so both are strictly smaller than the upper box dimension $2-s$.

In [6, p. 74], we find a result analogous to that of Theorem 3.2 , but it concerns wavelet series and is stated only in probabilistic form; however, we believe that a (deterministic) counterpart for Theorem 3.2 concerning wavelet series also holds.

REMARK 3.3. (a) Let $\left(\lambda_{j}\right)_{j \in \mathbb{N}} \subset \mathbb{R}^{+}$be such that the limit $\gamma:=$ $\lim _{j \rightarrow \infty} \lambda_{j+1} / \lambda_{j}$ exists and satisfies $\gamma>1$, and consider a convergent sequence $\left(s_{j}\right)_{j \in \mathbb{N}} \subset \mathbb{R}^{+}$such that $s:=\lim _{j \rightarrow \infty} s_{j}$ satisfies $0<s<1$. Let $W_{s}:[0,1] \rightarrow \mathbb{R}$ be defined by $W_{s}(x):=\sum_{j \geq 1} 2^{-\lambda_{j} s_{j}} g\left(2^{\lambda_{j}} x+\theta_{j}\right)$, where $\left(\theta_{j}\right)_{j \in \mathbb{N}} \subset \mathbb{R}$ and $g$ is as in Theorem 3.2. Then the identity of Theorem 3.2 remains true for these $\gamma$ and $s$ and this more general scale-sparse Weierstrasstype function.

(b) Consider $\rho>1,0<s<1,\left(\theta_{j}\right)_{j \in \mathbb{N}} \subset \mathbb{R}$, and $g$ as in Theorem 3.2 . Let $W_{s}:[0,1] \rightarrow \mathbb{R}$ be defined by $W_{s}(x):=\sum_{j \geq 1} \rho^{-j s} g\left(\rho^{j} x+\theta_{j}\right)$. Then (see [5, Theorem 8, p. 800]) we have

$$
\operatorname{dim}_{\mathrm{H}} \Gamma\left(W_{s}\right) \geq 2-s-\frac{c_{g, s}}{\log \rho}
$$

where $c_{g, s}$ does not depend on $\rho$; this inequality can also be proved by using the same techniques applied in the proof above, including the generalization 
when we replace the constant $s$ by any convergent sequence $\left(s_{j}\right)_{j \in \mathbb{N}} \subset \mathbb{R}^{+}$ such that $s:=\lim _{j \rightarrow \infty} s_{j}$ satisfies $0<s<1$.

4. Proofs of Theorem 3.2 and Remark 3.3. We first prove Theorem 3.2 and then use analogous arguments to prove Remark 3.3 .

Proof of Theorem 3.2. (i) Let us start by defining a Cantor-like set $K \subset$ $[0,1]$, in order to remove short open intervals around local maxima and minima of $W_{s}$, as follows. Let us denote by $K_{0}^{\prime}$ and $K_{0}^{\prime \prime}$ the two subintervals of monotonicity considered for $g$ in the theorem. Furthermore, we assume, without any loss of generality, that $g$ has period 1 , and that $K_{0}^{\prime}$ and $K_{0}^{\prime \prime}$ are closed congruent intervals, i.e. they have the same length.

Let $j(\rho, \gamma, s):=j\left(g, K_{0}^{\prime}, K_{0}^{\prime \prime}, \rho, \gamma, s\right)$ be a large positive number, and for each fixed integer $j \geq j(\rho, \gamma, s)$ consider the set $K_{j}$ of all $x \in[0,1]$ such that $\rho^{\gamma^{j}} x+\theta_{j} \in K_{0}^{\prime} \cup K_{0}^{\prime \prime}+k$ for some $k=k_{x} \in \mathbb{Z}$. Actually, we complete the definition of $K_{j}$ by removing from it all those $x$ for which $k_{x}$ is maximal or minimal over all $k$ taken (for $j$ fixed). Thus, $K_{j}$ is a union of congruent intervals in which $W_{j}(x):=g\left(\rho^{\gamma^{j}} x+\theta_{j}\right)$ satisfies, with alternating monotonicity on consecutive intervals, the growth properties assumed for $g$ on $K_{0}^{\prime}$ and $K_{0}^{\prime \prime}$.

So, $\bar{W}_{j}(x):=\sum_{j^{\prime}=1}^{j} \rho^{-\gamma^{j^{\prime}} s} g\left(\rho^{\gamma^{j^{\prime}}} x+\theta_{j^{\prime}}\right)$ has the same growth behavior on $\bar{K}_{j}:=\bigcap_{j^{\prime}=j(\rho, \gamma, s)}^{j} K_{j^{\prime}}$. Actually, we can write $\bar{K}_{j}$ also as a union of intervals $\bar{I}_{j}$ and, as before, we must complete the definition of $\bar{K}_{j}$ by removing from it all those intervals $\bar{I}_{j}$ which have length strictly smaller than most of them due to a truncation in the intersection above. In this way, we arrive at a (closed) Cantor-like set $K:=\bigcap_{j \geq j(\rho, \gamma, s)} \bar{K}_{j}$, which satisfies $\operatorname{dim}_{\mathrm{H}} K=1$.

In order to prove the last identity, we will deal with the most natural mass distribution, denoted here by $\mu_{K}$, supported on the Cantor set $K$ (cf. [3. pp. 13-14]). Using the Lebesgue measure $\delta:=\lambda\left(K_{0}^{\prime} \cup K_{0}^{\prime \prime}\right)$, we can obtain $\left|\bar{I}_{j}\right|=(\delta / 2) \rho^{-\gamma^{j}}$ and

$$
\mu_{K}\left(\bar{I}_{j}\right)=\left(2\left[\rho^{\gamma^{j(\rho, \gamma, s)}}\right]+\kappa_{j(\rho, \gamma, s)}\right)^{-1} \prod_{j^{\prime}=j(\rho, \gamma, s)+1}^{j}\left(2\left[\frac{(\delta / 2) \rho^{-\gamma^{j^{\prime}-1}}}{\rho^{-\gamma^{j^{\prime}}}}\right]+\kappa_{j^{\prime}}\right)^{-1},
$$

with $\kappa_{j^{\prime}} \in\{0, \pm 1, \pm 2\}$. This yields a result that will be useful in part (ii) below:

$$
\mu_{K}\left(\bar{I}_{j}\right)=\left|\bar{I}_{j}\right|^{d_{j}}, \quad \text { where } \quad \lim _{j \rightarrow \infty} d_{j}=1 .
$$

Considering now a ball $\dot{B}_{r} \subset \mathbb{R}$ centered at $X \in K$ and $r_{j+1}<r \leq r_{j}$ for some $j=j_{r}$ (where $r_{j} \approx_{j} \rho^{-\gamma^{j}}$ ), then we obtain $\mu_{K}\left(\dot{B}_{r}\right) \approx_{r} r_{j}^{d_{j}} r / r_{j} \leq c r^{d_{j}}$, and so the identity $\operatorname{dim}_{\mathrm{H}} K=1$ follows by Remark 2.3 . 
(ii) Now, we will prove formula (1) of the theorem. Invoking Theorem 4.1 of [2] and comparison between dimensions in Remark 2.3, we realize that is sufficient to prove that $\operatorname{dim}_{H} \Gamma\left(\left.W_{s}\right|_{K}\right) \geq 2-\Xi(\gamma, s)$. To do this, let us start by defining, induced by the mass distribution $\mu_{K}$ defined in part (i), and supported on the graph of $\left.W_{s}\right|_{K}$, the natural mass distribution

$$
\mu_{0}(U):=\mu_{\left.W_{s}\right|_{K}}(U):=\mu_{K}\left(\left\{x \in K:\left(x, W_{s}(x)\right) \in U\right\}\right)
$$

for Borel subsets $U$ of $\mathbb{R}^{2}$. In the rest of the proof, we will assume that $j \geq$ $j(\rho, \gamma, s)$ is an integer sufficiently large, according to part (i). We will estimate from above the mass of small balls centered at any point $P \in \Gamma\left(\left.W_{s}\right|_{K}\right)=$ supp $\mu_{0}$, considering first the balls $B_{j} \subset \mathbb{R}^{2}$ with diameter $\left|B_{j}\right|=r_{j} \approx_{j} \rho^{-\gamma^{j}}$ (see Figure 1 below). As we will see, these balls correspond asymptotically to the "minimal mass". On the other hand, in part (iii) below we will be in an opposite situation when we calculate the mass $\mu_{0}$ applied to appropriate balls $B_{j}^{\prime} \subset \mathbb{R}^{2}$ with diameter, according to Figure 1, given by the relation

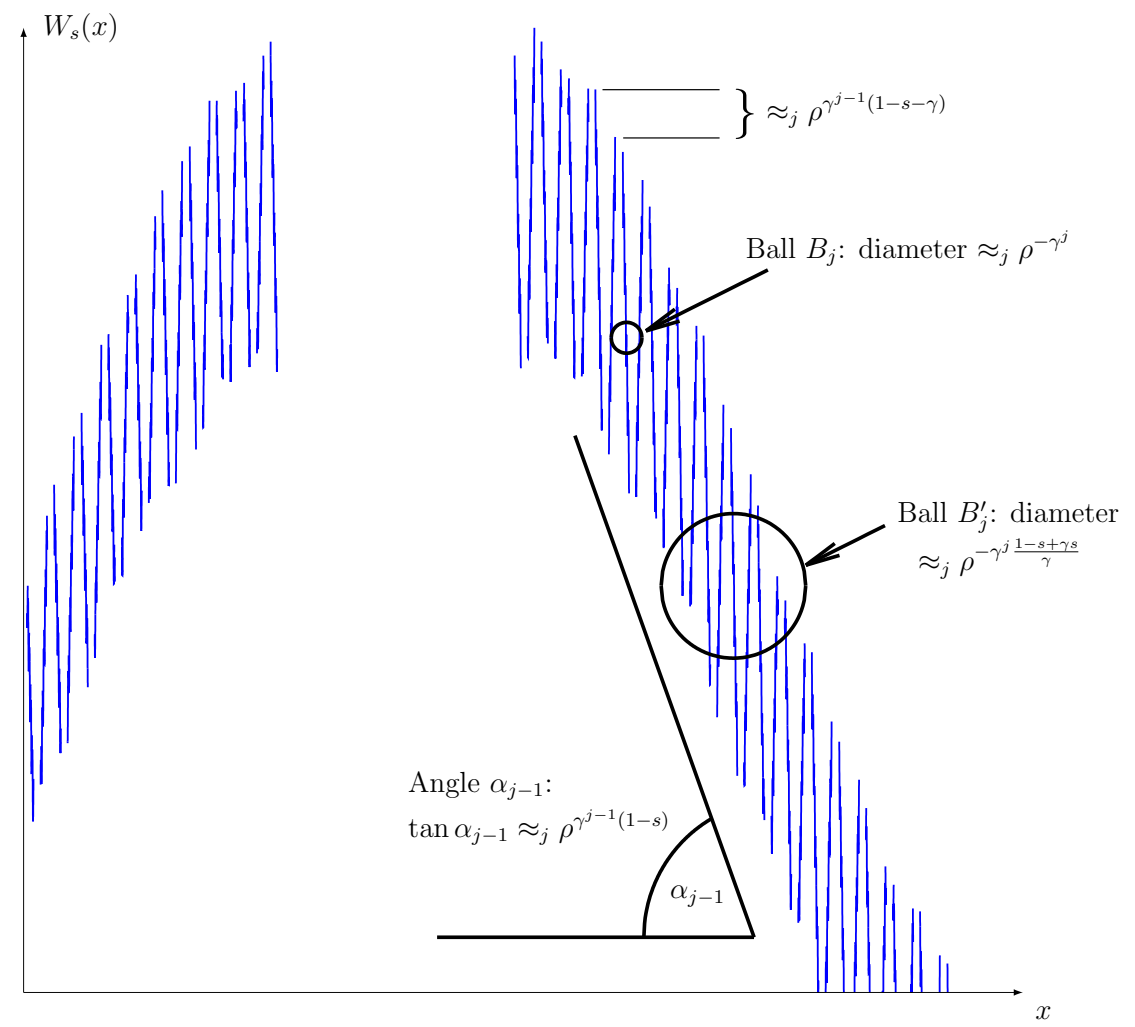

Fig. 1. Graph of the restriction $\left.W_{s}\right|_{K}$ to a Cantor-like set, according to the proof of Theorem 3.2 where $W_{s}(x)=\sum_{j \geq 1} \rho^{-\gamma^{j} s} g\left(\rho^{\gamma^{j}} x+\theta_{j}\right)$. 


$$
\left|B_{j}^{\prime}\right|=r_{j}^{\prime} \approx_{j} \rho^{-\gamma^{j} s} \cos \alpha_{j-1} \approx_{j} \rho^{-\gamma^{j} s} / \tan \alpha_{j-1} \approx_{j} \rho^{-\gamma^{j} s} / \rho^{\gamma^{j-1}(1-s)} .
$$

In fact, this class of balls with diameter $r_{j}^{\prime} \approx_{j} \rho^{-\gamma^{j-1}(1-s+\gamma s)} \approx_{j} r_{j-1}^{1-s+\gamma s} \approx_{j}$ $r_{j}^{(1-s+\gamma s) / \gamma}$ corresponds asymptotically to the "maximal mass", which allows us to obtain a sharp estimate for $\operatorname{dim}_{H} \Gamma\left(\left.W_{s}\right|_{K}\right)$. (We should observe that $1<1-s+\gamma s<\gamma$.)

Let $l \geq j(\rho, \gamma, s)$ be an integer and assume additionally, without any loss of generality, that $\min (g) \geq 0$ and $K_{0}^{\prime} \cup K_{0}^{\prime \prime} \subset[0,1]$. A scale peak of the function $\left.W_{s}\right|_{K}$, or, briefly, a peak, denoted by $\Lambda_{l}=\Lambda_{l}^{(m)}$, is by definition a subset of the graph of $\left.W_{s}\right|_{K}$, when restricted to $K \cap\left[\left(m-\theta_{l}\right) \rho^{-\gamma^{l}},\left(m+1-\theta_{l}\right) \rho^{-\gamma^{l}}\right]=$ $K \cap\left[\bar{I}_{l}^{\prime(m)} \cup \bar{I}_{l}^{\prime \prime(m)}\right]$ for some integer $m$ for which we have a non-empty intersection. Here, $\bar{I}_{l}^{\prime(m)}$ and $\bar{I}_{l}^{\prime \prime(m)}$ are two consecutive (congruent) intervals $\bar{I}_{l}$ of the set $\bar{K}_{l}$. The height of a peak $\Lambda_{l}, h_{\Lambda_{l}}$, obtained by elementary calculations, is given by $\approx_{l} \rho^{-\gamma^{l} s}$. More precisely,

$$
h_{\Lambda_{l}}:=\max _{\Lambda_{l}}-\min _{\Lambda_{l}} \approx_{l}\left|\sum_{j^{\prime} \geq l} \pm \rho^{-\gamma^{j^{\prime}} s}\right| \approx_{l} \rho^{-\gamma^{l} s}
$$

where $\min _{\Lambda_{l}}$ and $\max _{\Lambda_{l}}$ stand for the minimum and maximum, respectively, of the vertical coordinate over all points $\left(x,\left.W_{s}\right|_{K}(x)\right)$ of the peak $\Lambda_{l}$.

Moreover, most of the peaks $\Lambda_{l}$ have two geometrical halves, coming from the two monotonic parts of $\left.\bar{W}_{l}\right|_{\bar{I}_{l}^{\prime(m)} \cup \bar{I}_{l}^{\prime \prime(m)}}$, as can be graphically observed in Figure 1 where the two halves of a peak $\Lambda_{j-1}$ of $\left.W_{s}\right|_{K}$ are explicitly represented, as well as a large number of peaks $\Lambda_{j}$. For a given $j$, standard calculations show that both graphs of $\left.W_{s}\right|_{K}$ and $\left.\bar{W}_{j}\right|_{K}$ have geometrical peaks $\Lambda_{j}$ with height $\approx_{j} \rho^{-\gamma^{j} s}$, and that each peak $\Lambda_{j-1}$ contains $\approx_{j} \rho^{-\gamma^{j-1}} / \rho^{-\gamma^{j}}$ many peaks $\Lambda_{j}$.

Let us now estimate the mass $\mu_{0}=\mu_{\left.W_{s}\right|_{K}}$ of balls $B_{j-1}$ centered at any $P \in \Gamma\left(\left.W_{s}\right|_{K}\right)$, which are not explicitly represented in Figure 1 since they would appear too large. In order to do this, we define the angle $\alpha_{j-1}$ (see Figure 1) by the relation $\left|\bar{W}_{j-1}(y)-\bar{W}_{j-1}(x)\right| \approx_{j, x, y}|y-x| \tan \alpha_{j-1}$ on any of the intervals $\bar{I}_{j-1}$ of $\bar{K}_{j-1}$. Then

$$
\tan \alpha_{j-1} \approx_{j}\left|\sum_{j^{\prime}=1}^{j-1} \rho^{-\gamma^{j^{\prime}} s}\left( \pm \rho^{\gamma^{j^{\prime}}}\right)\right| \approx_{j} \rho^{\gamma^{j-1}(1-s)}
$$

on any interval $\bar{I}_{j-1}$, since $W_{j^{\prime}}(y)-W_{j^{\prime}}(x) \approx_{j^{\prime}, x, y} \pm \rho^{\gamma^{j^{\prime}}}(y-x)$ on each $\bar{I}_{j-1}$. By considering a fixed half $\Lambda^{*}$ of a peak $\Lambda_{j-1}$, and by taking into account the angle $\alpha_{j-1}$ on $\Lambda^{*}$, we can say that, roughly, the vertical position of a peak $\Lambda_{j}^{(m+1)}$ of $\Lambda^{*}$ is obtained from the one of the preceding peak $\Lambda_{j}^{(m)}$ by 
moving an "average distance" (see Figure 1)

$$
\sqsupset_{j}:=\rho^{-\gamma^{j}} \tan \alpha_{j-1} \approx_{j} \rho^{\gamma^{j-1}(1-s-\gamma)} .
$$

Let $C_{\Lambda^{*}}$ be the minimum of the vertical coordinate over all points $(x$, $\left.\left.\bar{W}_{j-1}\right|_{K}(x)\right)$ of the corresponding fixed half $\bar{\Lambda}^{*}$ for the function $\left.\bar{W}_{j-1}\right|_{K}$; then $C_{\Lambda^{*}}$ depends only on $\Lambda^{*}$. In precise terms, by taking into account the value of $\tan \alpha_{j-1}$ coming from the partial sum $\bar{W}_{j-1}$ on the interval $\bar{I}_{j-1}$ analogously to that coming from $W_{j-1}$ - as well as the height of every peak $\tilde{\Lambda}_{j}$ of $\left.W_{s}\right|_{K}-\left.\bar{W}_{j-1}\right|_{K}$, estimated by $\approx_{j} \rho^{-\gamma^{j} s}$ —analogously to that of a peak $\Lambda_{j}$ of $\left.W_{s}\right|_{K}$-we have the relation

$$
\min _{\Lambda_{j}^{(k)}}-C_{\Lambda^{*}} \approx_{j, k} k \sqsupset_{j}
$$

where the index $k \in \mathbb{N}_{0}$ establishes a rank on all peaks $\Lambda_{j}$ of the half $\Lambda^{*}$, by starting from the lowest vertical position with $k=0$. Because of this relation, and since a ball $B_{j-1}$ has height, or diameter, $\left|B_{j-1}\right|=r_{j-1} \approx_{j} \rho^{-\gamma^{j-1}}$, the number $N_{j}$ of peaks $\Lambda_{j}$ that intersect a given ball $B_{j-1}$ can be estimated from above by the quantity

$$
c \frac{\rho^{-\gamma^{j-1}}+h_{\Lambda_{j}}}{\sqsupset_{j}} \approx_{j} \frac{\rho^{-\gamma^{j-1}}+\rho^{-\gamma^{j} s}}{\rho^{\gamma^{j-1}(1-s-\gamma)}} .
$$

(ii1) Let $\gamma s \geq 1$. Hence $N_{j} \leq c \rho^{-\gamma^{j-1}} / \rho^{\gamma^{j-1}(1-s-\gamma)}=c \rho^{\gamma^{j-1}(-2+s+\gamma)}$. Therefore,

$$
\begin{aligned}
\mu_{0}\left(B_{j-1}\right) & \leq N_{j} \mu_{0}\left(\Lambda_{j}\right) \approx_{j} N_{j}\left(\rho^{-\gamma^{j}}\right)^{d_{j}} \\
& \leq c \rho^{-\gamma^{j-1}\left(2-s-\gamma+\gamma d_{j}\right)}=c \rho^{-\gamma^{j-1}\left(2-s-\varepsilon_{j}\right)},
\end{aligned}
$$

where $\varepsilon_{j}:=\gamma\left(1-d_{j}\right)$, thus (i) yields $\lim _{j \rightarrow \infty} \varepsilon_{j}=0$. So, $\mu_{0}\left(B_{j-1}\right) \leq$ $c\left|B_{j-1}\right|^{2-s-\varepsilon_{j}}$.

(ii2) Let $\gamma s \leq 1$ and define

$$
J:=\max \left\{j^{\prime} \in \mathbb{N}_{0}: \rho^{-\gamma^{j+j^{\prime}} s} \geq \rho^{-\gamma^{j-1}}\right\}=\max \left\{j^{\prime} \in \mathbb{N}_{0}: \gamma^{j^{\prime}} s \leq \gamma^{-1}\right\} .
$$

Because $\gamma s \leq 1$, for $l=j, \ldots, j+J$ we have

$$
\frac{\rho^{-\gamma^{j-1}}+h_{\Lambda_{l}}}{\sqsupset_{l}} \approx_{j, l} \frac{\rho^{-\gamma^{l} s}}{\rho^{\gamma^{l-1}(1-s-\gamma)}}=\rho^{\gamma^{l}(1-s)(1-1 / \gamma)} .
$$

This quantity is, up to a constant, an upper bound of the number of peaks $\Lambda_{l}$ which have height $\approx_{l} \rho^{-\gamma^{l} s}$, intersect a given ball $B_{j-1}$ and are contained in any of the two halves of a fixed peak $\Lambda_{l-1}$. So we have the inequality $N_{j} \leq c \rho^{\gamma^{j}(1-s)(1-1 / \gamma)}$ and, on the other hand, for each $l=j+1, \ldots, j+J+1$ the total number $N_{l}$ of peaks $\Lambda_{l}$ intersecting the fixed ball $B_{j-1}$ can be 
recursively estimated:

$$
\begin{aligned}
& N_{j+1} \leq c N_{j} \rho^{\gamma^{j+1}(1-s)(1-1 / \gamma)} \leq c^{\prime} \rho^{\gamma^{j}(1-s)(1-1 / \gamma)(1+\gamma)}, \\
& N_{j+2} \leq c N_{j+1} \rho^{\gamma^{j+2}(1-s)(1-1 / \gamma)} \leq c^{\prime} \rho^{\gamma^{j}(1-s)(1-1 / \gamma)\left(1+\gamma+\gamma^{2}\right)},
\end{aligned}
$$

and so on, arriving at the inequality

$$
N_{j+J} \leq c \rho^{\gamma^{j}(1-s)(1-1 / \gamma) \sum_{l=0}^{J} \gamma^{l}}=c \rho^{\gamma^{j}(1-s)\left(\gamma^{J+1}-1\right) / \gamma} .
$$

Here, we must observe that the number $J$ of iterations does not depend on $j$, so the product of the constants coming from the previous steps is also independent of $j$. Finally,

$$
\begin{aligned}
N_{j+J+1} & \leq c N_{j+J} \frac{\rho^{-\gamma^{j-1}}}{\rho^{\gamma^{(j+J+1)-1}(1-s-\gamma)}}=c N_{j+J} \rho^{-\gamma^{j}\left(1 / \gamma+\gamma^{J}(1-s-\gamma)\right)} \\
& \leq c^{\prime} \rho^{\gamma^{j}\left((1-s)\left(\gamma^{J}-1 / \gamma\right)-1 / \gamma-\gamma^{J}(1-s-\gamma)\right)}=c^{\prime} \rho^{\gamma^{j}\left((-2+s) / \gamma+\gamma^{J+1}\right)},
\end{aligned}
$$

and then we obtain

$$
\begin{aligned}
\mu_{0}\left(B_{j-1}\right) & \leq N_{j+J+1} \mu_{0}\left(\Lambda_{j+J+1}\right) \approx_{j} N_{j+J+1}\left(\rho^{-\gamma^{j+J+1}}\right)^{d_{j+J+1}} \\
& \leq c \rho^{-\gamma^{j}\left((2-s) / \gamma-\gamma^{J+1}+\gamma^{J+1} d_{j+J+1}\right)}=c \rho^{-\gamma^{j-1}\left(2-s-\varepsilon_{j}^{\prime}\right)},
\end{aligned}
$$

where $\varepsilon_{j}^{\prime}:=\gamma^{J+2}\left(1-d_{j+J+1}\right)$, thus by (i) we have $\lim _{j \rightarrow \infty} \varepsilon_{j}^{\prime}=0$. Hence, $\mu_{0}\left(B_{j-1}\right) \leq c\left|B_{j-1}\right|^{2-s-\varepsilon_{j}^{\prime}}$.

(ii3) From (ii1) and (ii2), we obtain for all $j$ the estimate (we remind the reader that $\left|B_{j}\right|=r_{j} \approx_{j} \rho^{-\gamma^{j}}$ )

$$
\mu_{0}\left(B_{j}\right) \leq c\left|B_{j}\right|^{2-s-\varepsilon_{j}}=c r_{j}^{2-s-\varepsilon_{j}}, \quad \text { where } \lim _{j \rightarrow \infty} \varepsilon_{j}=0 .
$$

Finally, in part (iii) below, we will easily find an estimate for the mass $\mu_{0}$ of the balls $B_{j}^{\prime}$ (see Figure 1 ) which have diameter

$$
\left|B_{j}^{\prime}\right|=r_{j}^{\prime} \approx_{j} \rho^{-\gamma^{j}(1-s+\gamma s) / \gamma}=r_{j}^{(1-s+\gamma s) / \gamma} .
$$

(iii) Taking into account the estimate obtained in part (ii) and the diameters of the balls $B_{j}$ and $B_{j}^{\prime}$, we get

$$
\mu_{0}\left(B_{j}^{\prime}\right) \leq c\left(r_{j}^{\prime} / r_{j}\right)^{2} \sup _{B_{j}} \mu_{0}\left(B_{j}\right) \leq c^{\prime}\left(r_{j}^{\prime}\right)^{2} r_{j}^{-s-\varepsilon_{j}} .
$$

Therefore,

$$
\mu_{0}\left(B_{j}^{\prime}\right) \leq c\left(r_{j}^{\prime}\right)^{2}\left(r_{j}^{\prime}\right)^{-\frac{\gamma\left(s+\varepsilon_{j}\right)}{1-s+\gamma s}}=c\left(r_{j}^{\prime}\right)^{2-\Xi(\gamma, s)-\varepsilon_{j}^{\prime}}, \quad \text { where } \quad \lim _{j \rightarrow \infty} \varepsilon_{j}^{\prime}=0 .
$$

Consider now a small ball $B_{r} \subset \mathbb{R}^{2}$ with $0<\left|B_{r}\right|=r<1$ and centered at any $P \in \Gamma\left(\left.W_{s}\right|_{K}\right)=\operatorname{supp} \mu_{0}$. Suppose first $r_{j} \leq r \leq r_{j}^{\prime}$ for some $j=j_{r}$. 
Then

$$
\begin{aligned}
\mu_{0}\left(B_{r}\right) & \leq c\left(r / r_{j}\right)^{2} \sup _{B_{j}} \mu_{0}\left(B_{j}\right) \leq c^{\prime} r^{2} r_{j}^{-s-\varepsilon_{j}} \\
& \approx_{r} c^{\prime} r^{2}\left(r_{j}^{\prime}\right)^{-\Xi(\gamma, s)-\varepsilon_{j}^{\prime}} \leq c^{\prime} r^{2-\Xi(\gamma, s)-\varepsilon_{j}^{\prime}} .
\end{aligned}
$$

Suppose now $r_{j}^{\prime} \leq r \leq r_{j-1}$ for some $j=j_{r}$. Then, by taking into account the particular characteristics of the graph of $\left.W_{s}\right|_{K}$ and the respective diameters of the balls,

$$
\mu_{0}\left(B_{r}\right) \leq c\left(r / r_{j}^{\prime}\right) \sup _{B_{j}^{\prime}} \mu_{0}\left(B_{j}^{\prime}\right) \leq c^{\prime} r\left(r_{j}^{\prime}\right)^{1-\Xi(\gamma, s)-\varepsilon_{j}^{\prime}} \leq c^{\prime} r^{2-\Xi(\gamma, s)-\varepsilon_{j}^{\prime}} .
$$

In this way we obtain the estimate $\mu_{0}\left(B_{r}\right) \leq c r^{2-\Xi(\gamma, s)-\varepsilon_{r}}$, where $\lim _{r \rightarrow 0^{+}} \varepsilon_{r}$ $=0$.

By Remark 2.3 we obtain $\operatorname{dim}_{\mathrm{H}} \Gamma\left(\left.W_{s}\right|_{K}\right) \geq 2-\Xi(\gamma, s)-\varepsilon$ for all $\varepsilon>0$, and by the comments at the beginning of part (ii), the proof is complete.

Proof of Remark 3.3. Part (a) follows from the proof of Theorem 3.2 with standard modifications. We will prove the inequality of part (b) by applying the same techniques we used in that proof. Likewise, we assume that $g$ has period $1, \min (g) \geq 0$, and $K_{0}^{\prime}, K_{0}^{\prime \prime} \subset[0,1]$ are congruent intervals. Additionally, we consider here $\rho \geq \rho(s):=\rho\left(g, K_{0}^{\prime}, K_{0}^{\prime \prime}, s\right)$ sufficiently large. Then standard calculations show that there are geometrical scale peaks in the graph of the function $\left.W_{s}\right|_{K}$, where $K \subset[0,1]$ is an appropriate Cantor-like set.

If we consider balls $\dot{B}_{j} \subset \mathbb{R}$ with diameter $\left|\dot{B}_{j}\right|=r_{j} \approx_{j} \rho^{-j}$, centered at any $X \in K=\operatorname{supp} \mu_{K}$, where $\mu_{K}$ is the mass distribution associated to the set $K$, then for all $j \in \mathbb{N}$,

$$
\mu_{K}\left(\dot{B}_{j}\right) \leq c r_{j}^{d_{j}} \quad \text { with } \quad d_{j}:=\frac{c^{\prime}+(j-1) \log (2[(\delta / 2) \rho]-2)}{c+(j-1) \log \rho},
$$

where the Lebesgue measure $\delta:=\lambda\left(K_{0}^{\prime} \cup K_{0}^{\prime \prime}\right)$ does not depend on $\rho$. Hence, by Remark 2.3 we have

$$
\operatorname{dim}_{\mathrm{H}} K \geq \lim _{j \rightarrow \infty} d_{j}=\frac{\log (2[(\delta / 2) \rho]-2)}{\log \rho}=1-\frac{c_{\delta, \rho}}{\log \rho},
$$

where $c_{\delta, \rho}:=\log \frac{\rho}{2[(\delta / 2) \rho]-2}$. (Observe that $c_{\delta, \rho} \rightarrow \log (1 / \delta)$ as $\rho \rightarrow \infty$.)

Let $0<\eta<1$ and $\rho(\eta):=\rho(\delta, \eta)$ be such that $c_{\delta, \rho} \leq c_{\delta, \eta}:=(1+\eta)$ $\log (1 / \delta)$ for all $\rho \geq \rho(\eta)$. In what follows consider $\rho \geq \rho(s, \eta):=\max \{\rho(s)$, $\rho(\eta)\}$ and $j \geq j(\rho, \eta, \delta)$ so large that $d_{j} \geq 1-c_{\delta, \eta} / \log \rho$.

We will estimate the mass $\mu_{0}$ of balls $B_{j-1} \subset \mathbb{R}^{2}$ centered at any $P \in$ $\Gamma\left(\left.W_{s}\right|_{K}\right)$, where $\mu_{0}(U):=\mu_{K}\left(\left\{x \in K:\left(x, W_{s}(x)\right) \in U\right\}\right)$ for Borel sets $U \subset \mathbb{R}^{2}$. We start by observing that, for fixed $j$, the vertical position of a peak $\Lambda_{j}^{(m+1)}$ is obtained from the position of the preceding peak $\Lambda_{j}^{(m)}$ by 
moving an "average distance"

$$
\approx_{j} \rho^{-j} \tan \alpha_{j-1} \approx_{j} \rho^{-j} \rho^{(j-1)(1-s)}=\rho^{-(1-s)} \rho^{-j s} .
$$

Because every peak $\Lambda_{j}$ has height $\approx_{j} \rho^{-j s}$, the number $N_{j}$ of peaks $\Lambda_{j}$ intersecting a given ball $B_{j-1}$ with height, or diameter, $\left|B_{j-1}\right|=r_{j-1} \approx_{j}$ $\rho^{-(j-1)}=c \rho^{-j}$ can be estimated from above by $c \frac{\rho^{-j}+\rho^{-j s}}{\rho^{-(1-s)} \rho^{-j s}} \approx_{j} 1$, therefore we can write $N_{j} \leq c$.

Set $J_{j}:=\max \left\{j^{\prime} \in \mathbb{N}_{0}: \rho^{-\left(j+j^{\prime}\right) s} \geq \rho^{-j}\right\}$, so $j-s \leq\left(j+J_{j}\right) s \leq j$. For $l=j+1, \ldots, j+J_{j}$, the number $N_{l}$ of peaks that intersect a given ball $B_{j-1}$ can be estimated recursively as

$$
N_{l} \leq c_{0}^{\prime} \frac{\rho^{-j}+\rho^{-l s}}{\rho^{-(1-s)} \rho^{-l s}} N_{l-1} \leq c_{0} \rho^{1-s} N_{l-1},
$$

therefore we finally obtain

$$
N_{j+J_{j}} \leq c\left(c_{0} \rho^{1-s}\right)^{J_{j}}=c c_{0}^{J_{j}} \rho^{J_{j}(1-s)} .
$$

(Observe that $c_{0}^{\prime}:=c_{0}^{\prime}\left(g, K_{0}^{\prime}, K_{0}^{\prime \prime}\right)$ and $c_{0}:=2 c_{0}^{\prime}$ depend only on $g, K_{0}^{\prime}, K_{0}^{\prime \prime}$.)

Unlike the proof of Theorem 3.2, part (ii2), here the number $J=J_{j}$ depends on $j$, so we take into account the inequalities $\frac{1-s}{s} j-1 \leq J_{j} \leq \frac{1-s}{s} j$, and the relations

$$
c_{0}^{J_{j}} \approx_{j} c_{s}^{j}=\left(\rho^{-j}\right)^{-\frac{\log c_{s}}{\log \rho}} \approx_{j}\left|B_{j-1}\right|^{-\frac{c_{s}^{\prime}}{\log \rho}},
$$

where $c_{s}:=c_{0}^{(1-s) / s}$ and $c_{s}^{\prime}:=\log c_{s}=\frac{1-s}{s} \log c_{0}$ (we can choose $c_{s}>1$, so $\left.c_{s}^{\prime}>0\right)$. Hence,

$$
\begin{aligned}
\mu_{0}\left(B_{j-1}\right) & \leq N_{j+J_{j}} \mu_{0}\left(\Lambda_{j+J_{j}}\right) \leq c N_{j+J_{j}}\left(\rho^{-\left(j+J_{j}\right)}\right)^{1-\frac{c_{\delta, \eta}}{\log \rho}-\varepsilon_{j}} \\
& \leq c^{\prime} c_{0}^{J_{j}} \rho^{-J_{j} s} \rho^{-j} \rho^{\left(j+J_{j}\right)\left(\frac{c_{\delta, \eta}}{\log \rho}+\varepsilon_{j}\right)} \approx_{j} c_{0}^{J_{j}} \rho^{-\left(j+J_{j}\right) s} \rho^{-j(1-s)} \rho^{j \frac{1}{s}\left(\frac{c_{\delta, \eta}}{\log \rho}+\varepsilon_{j}\right)} \\
& \approx_{j}\left|B_{j-1}\right|^{-\frac{c_{s}^{\prime}}{\log \rho}} \rho^{-j} \rho^{-j(1-s)} \rho^{j \frac{c_{\delta, \eta}}{\log \rho}} \rho^{j \frac{\varepsilon_{j}}{s}} \approx_{j}\left|B_{j-1}\right|^{2-s-\frac{c_{s}^{\prime}}{\log \rho}-\frac{c_{\delta, \eta}}{s \log \rho}-\frac{\varepsilon_{j}}{s}},
\end{aligned}
$$

where the quantity $\varepsilon_{j}:=1-c_{\delta, \eta} / \log \rho-d_{j+J_{j}}$ satisfies $\lim _{j \rightarrow \infty} \varepsilon_{j}=0$. Consequently, we have the estimate

$$
\mu_{0}\left(B_{r}\right) \leq c\left|B_{r}\right|^{2-s-\frac{c_{s}^{\prime}+c_{\delta, \eta} / s}{\log \rho}-\varepsilon_{r}} \quad \text { for all } 0<r<1
$$

where $\lim _{r \rightarrow 0^{+}} \varepsilon_{r}=0$. By Remark 2.3 we obtain

$$
\operatorname{dim}_{\mathrm{H}} \Gamma\left(\left.W_{s}\right|_{K}\right) \geq 2-s-\frac{c_{s}^{\prime}+c_{\delta, \eta} / s}{\log \rho}
$$

for all $\rho \geq \rho(s, \eta)=\max \{\rho(s), \rho(\eta)\}$, therefore we can complete the proof of part (b) by considering the quantity $c_{g, s}$ defined by $c_{g, s}:=\max \left\{c_{s}^{\prime}+\right.$ $\left.c_{\delta, \eta} / s,(1-s) \log \rho(s, \eta)\right\}$. 
OBSERVATION. As we just have the estimate $2-s-c_{g, s} / \log \rho \leq \operatorname{dim}_{\mathrm{H}} \Gamma\left(W_{s}\right)$ $\leq 2-s$-as mentioned before, the second (last) inequality is well-known - it is of interest to minimize the number $c_{g, s}$.

For fixed $g, \rho, s$ and $\eta$, we observe the following concerning $c_{g, s}$ : When $\delta$ increases, the values of $c_{\delta, \eta}$ and of $\rho(\eta)$ decrease, but the values of $\rho(s)$ and of $c_{s}^{\prime}$ may increase, so we have a trade-off when intending to minimize $c_{g, s}$.

Therefore, even knowing that $\operatorname{dim}_{\mathrm{H}} \Gamma\left(W_{s}\right)=2-s$ holds in many casessee e.g. Theorem 3.1 in general we do not know the actual value of $\operatorname{dim}_{H} \Gamma\left(W_{s}\right)$ up to an (apparently unavoidable) additive constant given by $c_{g, s} / \log \rho$.

Acknowledgments. I would like to acknowledge the important contributions made by Prof. Caetano, who read carefully all preliminary versions of the paper and gave many valuable comments for improvement and enrichment of the text. I would also like to thank Prof. Triebel for sharing his precious experience with me; finally, grateful thanks to both of them for suggesting the way forward in my research, always in a perfect atmosphere.

This research was partially supported by Gabinete de Relações Internacionais da Ciência e do Ensino Superior and by Deutscher Akademischer Austauschdienst (Covenant GRICES-DAAD) and by the Junior Research Team 'Fractal Analysis' of the University of Jena.

It was also partially supported by Unidade de Investigação Matemática e Aplicações of Universidade de Aveiro through Programa Operacional 'Ciência, Tecnologia, Inovação' (POCTI) of the Fundação para a Ciência e a Tecnologia (FCT), cofinanced by the European Community Fund (FEDER).

The author also acknowledges the support of the Fundação para a Ciência e a Tecnologia (FCT) and the European Social Fund in the scope of Community Support Framework III (Grant number SFRH/BD/16029/2004).

\section{References}

[1] A. S. Besicovitch and H. D. Ursell, Sets of fractional dimensions (V): On dimensional numbers of some continuous curves, J. London Math. Soc. 32 (1937), 142-153.

[2] A. Carvalho, Fractal geometry of Weierstrass-type functions, Fractals 17 (2009), $23-37$.

[3] K. Falconer, Fractal Geometry, Wiley, Chichester, 1990.

[4] B. R. Hunt, The Hausdorff dimension of graphs of Weierstrass functions, Proc. Amer. Math. Soc. 126 (1998), 791-800.

[5] R. Mauldin and S. Williams, On the Hausdorff dimension of some graphs, Trans. Amer. Math. Soc. 298 (1986), 793-803.

[6] F. Roueff, Dimension de Hausdorff du graphe d'une fonction continue: une étude analytique et statistique, $\mathrm{PhD}$ thesis, École Nationale Supérieure des Télécommunications, 2000. 
[7] H. Triebel, Fractals and Spectra, Birkhäuser, Basel, 1997.
Abel Carvalho
Departamento de Matemática
Universidade de Aveiro
3810-193 Aveiro, Portugal
E-mail: abel@mat.ua.pt

Received 25 November 2008;

in revised form 31 December 2010 
